\title{
openheart Repetitive squatting exercise as a diagnostic predictor in patients with dilated cardiomyopathy
}

Krunoslav Michael Sveric, Stefan Ulbrich, Mohamed Rady, Christian Pflücke, Silvio Quick, Stefanie Katzke, Karim Ibrahim, Ruth H Strasser, Stefanie Jellinghaus

\begin{abstract}
- Additional material is published online only. To view please visit the journal online (http://dx.doi.org/10.1136/ openhrt-2017-000733).
\end{abstract}

To cite: Sveric KM, Ulbrich S, Rady M, et al. Repetitive squatting exercise as a diagnostic predictor in patients with dilated cardiomyopathy. Open Heart 2018;5: 000733. doi:10.1136/

openhrt-2017-000733

KMS and SU contributed equally.

Received 5 October 2017 Revised 20 December 2017 Accepted 29 December 2017
Check for updates

Department for Internal Medicine and Cardiology, Herzzentrum Dresden, University Clinic, Technische Universität Dresden, Dresden, Germany

\section{Correspondence to}

Dr. Krunoslav Michael Sveric, Department of Cardiology, TU Dresden, Dresden 01307, Germany; kruno.sveric@ caroconnect.de

\section{ABSTRACT}

Objectives Non-ischaemic dilated cardiomyopathy (DCM) is characterised by a highly variable disease progression. Stress echocardiography and cardiopulmonary exercise testing (CPET) are beneficial in risk assessment, but are labour intensive. Repetitive squatting and standing without weights is a simple exercise (EX). The aim of this study was to investigate the prognostic role of left ventricular (LV) contractile recruitment (CR) after a simple EX of repetitive squatting through three-dimensional (3D) echocardiography.

Methods Patients with DCM (LV ejection fraction $(\mathrm{EF})<50 \%, \mathrm{n}=68$ ) and age-matched healthy volunteers $(n=25)$ received a $3 D$ echocardiographic evaluation of $L V$ EF before and after 30 repetitions of squatting-standing EX. CR was defined by the change of LV EF $(\Delta>4 \%)$. Patients were followed up prospectively (2 years) for cardiac death and deteriorating heart failure.

Results During follow-up, 14 cardiac events occurred (21\%) with six deaths and eight severe heart failure deteriorations. A poor CR after squatting EX differentiated DCM patients with cardiac events during follow-up as accurately as a reduced peak oxygen consumption (peak V0 ${ }_{2}<20 \mathrm{~mL} / \mathrm{kg} / \mathrm{min}$ ) (sensitivity: 0.97 and 0.95). Both had a significant incremental diagnostic value over clinical (age, dyspnoea and natriuretic peptide level) or resting echocardiographic parameters (E/E' ratio, LV EF and end-diastolic LV volume) to predict cardiac events (global $\chi^{2}: 16.0$ vs $5.3 ; 19.5$ vs 6.1 ; $\mathrm{P}<0.01$ for all).

Conclusions The presence of LV CR after EX of repetitive squatting without weights can stratify risk and predict cardiac events in patients with DCM as correct as CPET.

\section{INTRODUCTION}

Non-ischaemic dilated cardiomyopathy (DCM) is characterised by a progressive left ventricular (LV) chamber dilatation and global myocardial dysfunction. Its clinical course varies considerably among patients with mild symptoms and with similarly reduced cardiac function at rest. Stress echocardiography and cardiopulmonary exercise testing (CPET) have evolved as valuable tools for differentiating unfavourable prognoses in patients with systolic heart failure. ${ }^{1-4}$ Stress echocardiography assess directly LV

\section{Key messages}

What is already known about this subject? Measurement of left ventricular contractile reserve by stress echocardiography and functional capacity by cardiopulmonary exercise testing are superior determinants of disease progression in patients with non-ischaemic dilated cardiomyopathy than left ventricular ejection fraction at rest. However, both methods are labour intensive and not accessible in daily practice.

What does this study add?

Determination of left ventricular contractile reserve by simple exercise of repetitive squatting without weights predicts cardiac events about as functional capacity assessed by cardiopulmonary exercise testing in patients with non-ischaemic dilated cardiomyopathy.

How might this impact on clinical practice? These findings have the potential to enrich the daily armamentarium of cardiologists revealing the crucial difference between heart failure patients with apparently similar stable clinical conditions at high risk and those at low midterm risk.

contractile reserve (CR) measuring myocardial viability, which is achieved by an infusion of dobutamine or by physical exercise (EX). ${ }^{5}$ Peak oxygen consumption (peak $\mathrm{VO}_{2}$ ) during CPET relates to peak cardiac output and reflects patient's functional capacity. ${ }^{6}$ Stress echocardiography and CPET are safe methods, but they are labour intensive, which is why patient therapy is most commonly based on two-dimensional (2D) echocardiographic measurements of resting LV ejection fraction (EF) in an ambulatory setting. In contrast, LV chamber dimensions and EFs obtained by three-dimensional (3D) echocardiography show good agreement with measurements from cardiac MRI and are superior to the results of the $2 \mathrm{D}$ technique. ${ }^{7-9}$ Furthermore, 3D echocardiographic imaging does not rely on geometric assumptions and enables efficient image acquisition in one 


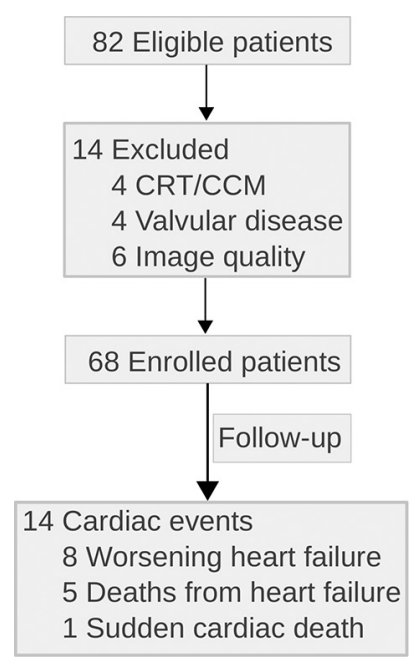

Figure 1 Flowchart representing the selection process of the study and the type of cardiac events occurring during follow-up period. CCM, cardiac contractility modulation; $\mathrm{CRT}$, cardiac resynchronisation therapy.

data set without repositioning of the ultrasound probe. These advantages of 3D echocardiography are desirable when cardiac function is expected to recuperate within minutes post EX. ${ }^{9}$

Repetitive squatting and standing without weights is a feasible EX that engages multiple muscle groups. As a dynamic EX, it would exhibit positive inotropic and chronotropic effects. ${ }^{10}$ Thus, the aim of this study was to investigate the prognostic role of LV CR after repetitive squatting EX by means of 3D echocardiography in patents with DCM.

\section{METHODS \\ Study population}

Eighty two clinically stable outpatients with a recent ( $<6$ months) diagnosis of non-ischaemic DCM with good acoustic window but without orthopaedic disorders were screened for this prospective study. Inclusion criteria were as follows: (1) LV EF $<50 \%$ in the absence of a coronary disease, (2) no clinical or magnetic-tomographic signs of myocarditis, (3) sinus rhythm without typical left bundle branch block and (4) full medical treatment according to the European heart failure guidelines. ${ }^{11}$ Right from the beginning, patients with cardiac resynchronisation/ modulation therapy and with more than moderate-to-severe valve diseases were excluded $(\mathrm{n}=8)$ (figure 1$)$. Twenty-five age and sex-matched healthy volunteers without a history of cardiovascular disease were included as controls. None of them reported regular training habits or had any clinical or anamnestic evidence of arterial hypertension or other cardiovascular diseases. All controls had an echocardiographic LV EF $>50 \%$, without significant valvular disease. This study was approved by the institutional review board, and written informed consent was obtained from all subjects.

\section{Study protocol}

On study enrolment, all subjects received a full 2D echocardiographic examination at rest as described previously, ${ }^{12}$ and their N-terminal pro-B-type natriuretic peptide (NT-proBNP) plasma levels were measured. The 3D echocardiographic examinations were performed at rest and within the first 2 min after 1 set of a maximum of 30 repetitions of squatting. This number of

\begin{tabular}{|c|c|c|c|c|c|}
\hline & $\begin{array}{l}\text { Controls } \\
(n=25)\end{array}$ & $\begin{array}{l}\text { All patients } \\
(\mathrm{n}=68)\end{array}$ & $\begin{array}{l}\text { Without CR } \\
(n=39)\end{array}$ & $\begin{array}{l}\text { With CR } \\
(n=29)\end{array}$ & P value* \\
\hline Age, years & $46 \pm 11$ & $50 \pm 11$ & $50 \pm 11$ & $49 \pm 11$ & 0.55 \\
\hline Male sex, n (\%) & $22(89)$ & $60(88)$ & $35(90)$ & $25(87)$ & 0.85 \\
\hline $\mathrm{BSA}, \mathrm{m}^{2}$ & $1.86 \pm 0.11$ & $1.98 \pm 0.18$ & $1.97 \pm 0.17$ & $1.99 \pm 0.17$ & 0.81 \\
\hline Systolic BP, mm Hg & $125 \pm 10$ & $123 \pm 19$ & $125 \pm 20$ & $121 \pm 17$ & 0.44 \\
\hline Diastolic BP, mm Hg & $61 \pm 7$ & $71 \pm 12$ & $73 \pm 14$ & $70 \pm 11$ & 0.47 \\
\hline NT-proBNP, pg/mL & / & $532(200: 1267)$ & $533(249: 684)$ & $525(191: 1400)$ & 0.78 \\
\hline NYHA functional class, n(\%) & & & & & 0.09 \\
\hline 1 & / & $36(53)$ & $16(41)$ & $20(69)$ & \\
\hline ॥ & I & $25(37)$ & $18(46)$ & $7(24)$ & \\
\hline III & / & $7(10)$ & $5(13)$ & $2(7)$ & \\
\hline \multicolumn{6}{|l|}{ Medication, $\mathrm{n}(\%)$} \\
\hline$\beta$-Blockers & / & $63(92)$ & $36(92)$ & 27 (93) & 0.99 \\
\hline AAl & I & $65(95)$ & $37(94)$ & $28(96)$ & 0.99 \\
\hline Diuretics & I & $54(80)$ & $33(84)$ & $22(75)$ & 0.58 \\
\hline
\end{tabular}

Values are mean with SD $( \pm)$ or absolute numbers with relative frequencies (\%).

${ }^{*}$ Patients with versus without contractile reserve assessed with appropriate test.

AAl, inhibitors of ACE or angiotensin I receptors; BP, blood pressure; BSA, body surface area; CR, contractile reserve; NT-proBNP, N-terminal pro-B-type natriuretic peptide shown as median (IQR); NYHA, New York Heart Association. 
Table 2 Echocardiographic characteristics before and after squatting EX

\begin{tabular}{|c|c|c|c|c|c|}
\hline & $\begin{array}{l}\text { Controls } \\
(\mathrm{n}=25)\end{array}$ & $\begin{array}{l}\text { All patients } \\
(n=68)\end{array}$ & $\begin{array}{l}\text { Without CR } \\
(n=39)\end{array}$ & $\begin{array}{l}\text { With CR } \\
(n=29)\end{array}$ & P value * \\
\hline $\mathrm{E} / \mathrm{E}^{\prime}$ ratio at rest & $7 \pm 3$ & $12 \pm 3$ & $12 \pm 3$ & $11 \pm 4$ & 0.81 \\
\hline \multicolumn{6}{|l|}{$\mathrm{EDV}, \mathrm{mL} / \mathrm{m}^{2}$} \\
\hline At rest & $67 \pm 11$ & $102 \pm 27$ & $105 \pm 29$ & $99 \pm 23$ & 0.32 \\
\hline After EX & $67 \pm 13$ & $104 \pm 30$ & $110 \pm 33 \dagger$ & $98 \pm 25$ & 0.09 \\
\hline$\Delta$ (absolute) & $0 \pm 5$ & $2 \pm 11$ & $5 \pm 12$ & $-1 \pm 11$ & 0.03 \\
\hline \multicolumn{6}{|l|}{$\mathrm{ESV}, \mathrm{mL} / \mathrm{m}^{2}$} \\
\hline At rest & $32 \pm 6$ & $71 \pm 25$ & $75 \pm 25$ & $67 \pm 24$ & 0.19 \\
\hline After EX & $26 \pm 8 \dagger$ & $70 \pm 30$ & $81 \pm 30 \dagger$ & $57 \pm 24 \dagger$ & $<0.001$ \\
\hline$\Delta$ (absolute) & $-6 \pm 5$ & $-1 \pm 12$ & $6 \pm 11$ & $-10 \pm 8$ & $<0.001$ \\
\hline \multicolumn{6}{|l|}{$E F, \%$} \\
\hline At rest & $56 \pm 3$ & $32 \pm 10$ & $31 \pm 10$ & $34 \pm 10$ & 0.12 \\
\hline After EX & $63 \pm 7 \dagger$ & $35 \pm 12 \dagger$ & $29 \pm 11 \dagger$ & $43 \pm 10 \dagger$ & $<0.001$ \\
\hline$\Delta$ (absolute) & $7 \pm 3$ & $3 \pm 7$ & $-2 \pm 4$ & $9 \pm 4$ & $<0.001$ \\
\hline \multicolumn{6}{|l|}{$\mathrm{SV}, \mathrm{mL} / \mathrm{m}^{2}$} \\
\hline At rest & $36 \pm 6$ & $31 \pm 9$ & $31 \pm 10$ & $32 \pm 8$ & 0.54 \\
\hline After EX & $42 \pm 7 \dagger$ & $34 \pm 10 \dagger$ & $29 \pm 8$ & $41 \pm 8 \dagger$ & $<0.001$ \\
\hline$\Delta$ (absolute) & $6 \pm 2$ & $3 \pm 8$ & $-2 \pm 6$ & $9 \pm 7$ & $<0.001$ \\
\hline \multicolumn{6}{|l|}{$\mathrm{Cl}, \mathrm{L} / \mathrm{min} / \mathrm{m}^{2}$} \\
\hline At rest & $2.7 \pm 0.7$ & $2.4 \pm 0.8$ & $2.5 \pm 0.9$ & $2.3 \pm 0.7$ & 0.32 \\
\hline After EX & $4.3 \pm 0.9 \dagger$ & $3.3 \pm 1.4 \dagger$ & $2.9 \pm 0.8 \dagger$ & $3.8 \pm 1.2 \dagger$ & 0.008 \\
\hline$\Delta$ (absolute) & $1.6 \pm 0.4$ & $0.9 \pm 1.2$ & $0.4 \pm 0.6$ & $1.5 \pm 0.9$ & $<0.001$ \\
\hline \multicolumn{6}{|l|}{ Heart rate, $1 / \min$} \\
\hline At rest & $71 \pm 7$ & $79 \pm 17$ & $81 \pm 18$ & $77 \pm 14$ & 0.43 \\
\hline After EX & $111 \pm 25 \dagger$ & $99 \pm 25 \dagger$ & $103 \pm 24 \dagger$ & $94 \pm 26 \dagger$ & 0.11 \\
\hline$\Delta$ (absolute) & $40 \pm 8$ & $19 \pm 17$ & $19 \pm 16$ & $19 \pm 17$ & 0.93 \\
\hline \multicolumn{6}{|c|}{ Systolic BP, mm Hg } \\
\hline At rest & $120 \pm 6$ & $114 \pm 18$ & $116 \pm 20$ & $112 \pm 17$ & 0.43 \\
\hline After EX & $156 \pm 14$ & $155 \pm 35 \dagger$ & $156 \pm 40 \dagger$ & $153 \pm 19 \dagger$ & 0.52 \\
\hline$\Delta$ (absolute) & $36 \pm 6$ & $30 \pm 25$ & $27 \pm 27$ & $26 \pm 19$ & 0.42 \\
\hline
\end{tabular}

Mean and SD ( \pm ).

${ }^{*}$ As in table 1.

$\dagger \mathrm{P}<0.01$ between rest and after exercise assessed with appropriate test.

$\mathrm{BP}$, blood pressure; $\mathrm{Cl}$, cardiac index; $\mathrm{CR}$, contractile reserve; $\mathrm{E} / \mathrm{E}^{\prime}$, ratio of pulsed-wave Doppler-derived early diastolic transmitral flow and tissue Doppler-derived early diastolic velocity obtained from the lateral mitral annulus; EDV, end-diastolic volume; EF, ejection fraction; ESV, end-systolic volume; EX, exercise; SV, stroke volume.

squatting repetitions is considered a fair to good performance in healthy middle-aged subjects. ${ }^{13}$ CPET followed separately within 2 days without a change of medical regime. The primary outcome was defined as composite end point of death or hospitalisation due to deteriorating heart failure, sudden cardiac death or ventricular arrhythmias requiring hospital admission. Follow-up was performed by cardiologists blinded to the results previously obtained from repetitive squatting EX. No patient was lost during follow-up (figure 1).

\section{Image acquisition and repetitive squatting EX}

Apical 3D echocardiography of the left ventricle was obtained using a PST-25SX (1-4 MHz) phased-array matrix transducer (Toshiba Artida 4D system, Japan) in left cubital position at rest and again in left cubital position immediately within $2 \mathrm{~min}$ after completion of repetitive squatting EX. Blood pressure was measured at rest and immediately after repetitive squatting EX. ECG-gated subvolumes of four to six heart cycles were acquired during a breath-holding manoeuvre. Elevation tilt, lateral contrast and volume rates (mean $22 \pm 3$ videos per second) were adjusted to achieve optimum imaging quality. Six patients $(8 \%)$ were excluded from follow-up analysis because of poor image quality after repetitive squatting EX.

\section{Image analysis}

All data sets of 3D echocardiographic LV volumes were analysed offline semi-automatically by a dedicated wall 
Table 3 Cardiopulmonary test results of patients without and with a CR

\begin{tabular}{|c|c|c|c|c|}
\hline & All patients $(n=68)$ & Without CR $(n=39)$ & With CR $(n=29)$ & P value* \\
\hline \multicolumn{5}{|l|}{ Peak workload } \\
\hline Watt & $139 \pm 43$ & $131 \pm 41$ & $147 \pm 44$ & 0.19 \\
\hline \multicolumn{5}{|l|}{ Heart rate, $1 / \mathrm{min}$} \\
\hline At rest & $78 \pm 15$ & $82 \pm 17$ & $76 \pm 13$ & 0.45 \\
\hline Peak & $132 \pm 32 \dagger$ & $130 \pm 34 \dagger$ & $134 \pm 31 \dagger$ & 0.72 \\
\hline \multicolumn{5}{|l|}{ Systolic BP, mm Hg } \\
\hline At rest & $124 \pm 18$ & $126 \pm 20$ & $122 \pm 17$ & 0.43 \\
\hline Peak & $160 \pm 35 \dagger$ & $160 \pm 40 \dagger$ & $161 \pm 31 \dagger$ & 0.92 \\
\hline \multicolumn{5}{|l|}{ Diastolic BP, mm Hg } \\
\hline At rest & $72 \pm 13$ & $72 \pm 14$ & $71 \pm 10$ & 0.61 \\
\hline Peak & $80 \pm 16$ & $79 \pm 20$ & $82 \pm 10$ & 0.52 \\
\hline Peak VO ${ }_{2}, \mathrm{~mL} / \mathrm{Kg} / \mathrm{min}$ & $22 \pm 7$ & $18 \pm 6$ & $25 \pm 7$ & 0.002 \\
\hline RER & $1.07 \pm 0.13$ & $1.06 \pm 0.14$ & $1.08 \pm 0.13$ & 0.74 \\
\hline
\end{tabular}

Values are mean with SD $( \pm)$.

${ }^{*}$ As in table 1.

$\dagger \mathrm{P}<0.01$ between rest and peak exercise assessed with appropriate test.

$\mathrm{BP}$, blood pressure; $\mathrm{CR}$, contractile reserve; peak $\mathrm{VO}_{2}$, peak oxygen consumption; RER, respiratory exchange ratio.

motion tracking software (WMT, Toshiba Medical System, Japan) as described previously. ${ }^{7}$ Three independent measures were performed and represented as the individual's mean. $\mathrm{LV}$ end-diastolic volume (EDV) and $\mathrm{LV}$ end-systolic volume (ESV) derived from 3D echocardiography were indexed to body surface area and used to calculate stroke volume (SV), cardiac index (CI) and LV EF. Intra-observer reliability of EDV, ESV and LV EF was estimated from 15 randomly selected data sets at rest and after repetitive squatting EX by the same investigator at least 1 week apart.

\section{Cardiopulmonary exercise testing}

All patients underwent a symptom-limited bicycle CPET based on three distinct stages under medical supervision until exhaustion. ${ }^{14}$ Blood pressure values and a continuous 12-lead ECG were recorded. Peak $\mathrm{VO}_{2}$ was measured, and the respiratory exchange ratio indicated the patient's effort during CPET.

\section{Statistical analysis}

Peak $\mathrm{VO}_{2}<20 \mathrm{~mL} / \mathrm{kg} / \mathrm{min}$ was defined as a reduced functional capacity. ${ }^{15}$ Differences in echocardiographic parameters between rest and after squatting EX were expressed as $\Delta$ values. Patients were divided into two groups, based on whether they developed a CR with $\Delta$ LV EF $>4 \%$ or not. ${ }^{16}$ Continuous data were presented as mean (with SD) unless otherwise specified. Normal distribution was evaluated by the Shapiro-Wilk W test. Comparisons between groups' mean and between rest versus after repetitive squatting EX were performed using paired/unpaired Student's t-test or Wilcoxon signed-rank test as appropriate. Pearson's correlation coefficient (r) was assessed. Categorical data were summarised as frequencies and percentages and were subjected to Fisher's exact test. Univariate Cox proportional hazard analysis was applied to identify predictors of the primary end point. Effect sizes of predictors were expressed as HR with 95\% CI (see table 4). Predictors from univariate regression with $\mathrm{P}<0.10$ or important clinical markers were used for multivariate Cox analyses as summarised in online TableS1 of supplementary material. In detail, basal regression models with standard clinical (ie, age, New York Heart Association (NYHA) and NT-proBNP) or standard echocardiographic (ie, age, E/E' ratio and resting LV EF) parameters were constructed. These models were augmented to predict cardiac events by addition of peak $\mathrm{VO}_{2}$ from CPET or/and CR from repetitive squatting EX (ie, $\Delta \mathrm{LV} E F$ ). These Cox models were separately compared by likelihood ratio tests expressed as global $\chi^{2}$ values with the corresponding significance value indicating higher predictive power for events during follow-up (figure 3). Final statistical significance was defined as a two-tailed $P$ value $<0.05$. All statistical analyses were performed with open-source software R (V.3.0.2, 2013, The R Foundation for Statistical Computing, Vienna).

\section{RESULTS}

\section{Baseline characteristics at study enrolment}

Table 1 summarises the anthropomorphic and clinical characteristics of patients with DCM and healthy controls at the time of study enrolment. Clinical characteristics did not differ significantly between DCM patients with CR and without CR, except for a trend of higher NYHA functional class and ESV in patients without CR. Both patient groups received equal medical treatment.

\section{LV function after repetitive squatting EX at baseline}

Comparisons of echocardiographic and haemodynamic data according to the presence or absence of CR are 

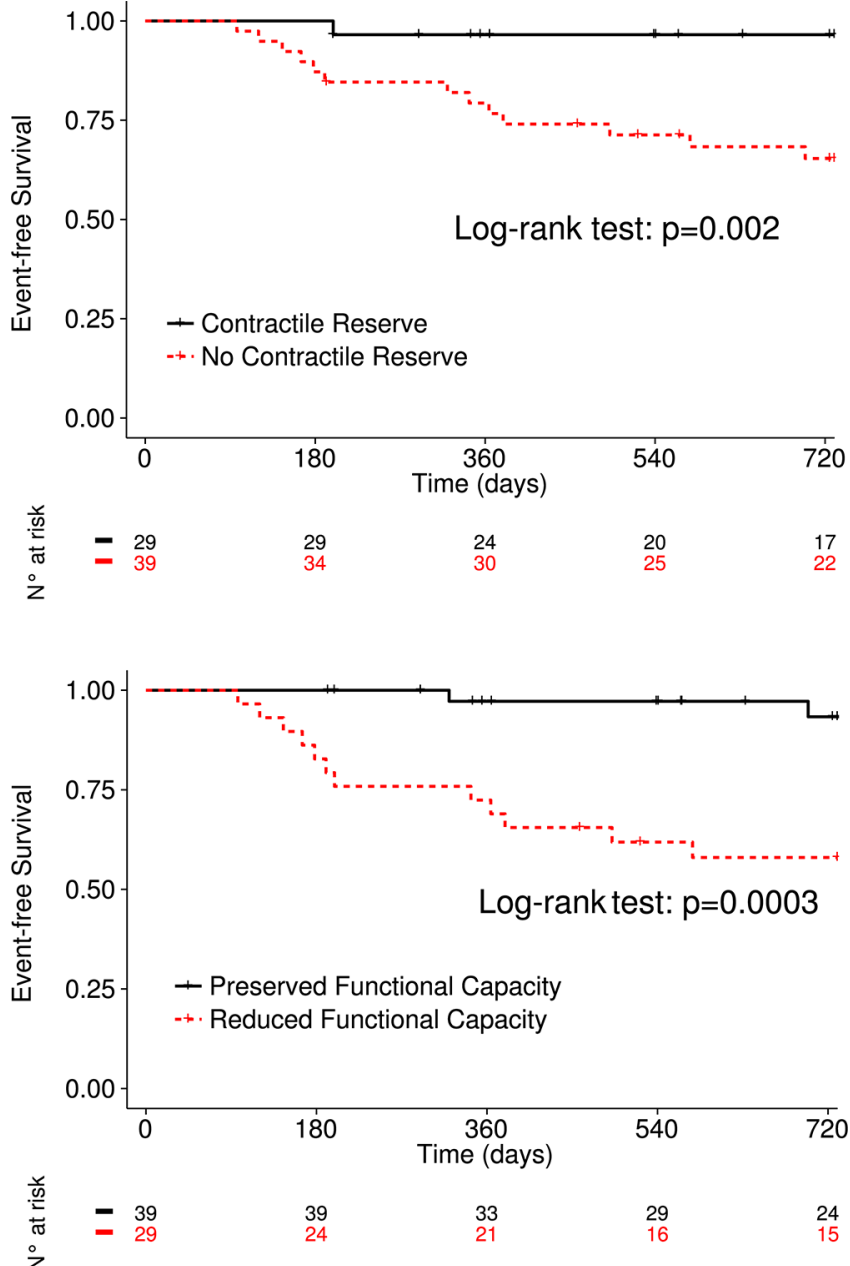

Figure 2 Event-free survival in patients with dilated cardiomyopathy stratified by the existence of contractile reserve ( $\Delta$ left ventricula ejection fraction $>4 \%$ ) after repetitive squatting exercise (upper) or by the existence of preserved functional capacity (peak oxygen consumption $>20 \mathrm{~mL} / \mathrm{Kg}$ / min) from cardiopulmonary exercise testing (lower).

reported in table 2. Interestingly, there were no significant echocardiographic differences between patients with $\mathrm{CR}$ and without CR at rest. However, patients without a CR showed not only an inadequate increase of LV EF, SV and CI but a rise of ESV and EDV after squatting EX. In contrast, patients with a CR exhibited an overall increase of LV functional parameters with a proportional decrease of ESV, while EDV remained stable after squatting EX. These characteristics were similar to healthy controls. Of note, $\triangle \mathrm{LV}$ EF ranged from $4 \%$ to $12 \%$ in healthy controls. $\triangle \mathrm{LV}$ EF showed strong correlation with $\triangle \mathrm{LV}$ ESV, $\Delta \mathrm{SV}$ and $\Delta$ CI after repetitive squatting $\mathrm{EX}(\mathrm{r}=-0.75,0.79$ and $0.67, \mathrm{P}<0.001$ for all), modest correlation with $\Delta \mathrm{LV}$ $\operatorname{EDV}(\mathrm{r}=-0.30 ; \mathrm{P}=0.01)$ and no significant association was found with $\mathrm{E} / \mathrm{E}^{\prime}$ ratio, age or medication $(\mathrm{P}>0.05$ for all).

\section{CPET at baseline}

At study enrolment, peak $\mathrm{VO}_{2}$ was significantly lower in patients without $\mathrm{CR}$ than in those with $\mathrm{CR}$, although workload, EX effort and haemodynamics at peak CPET
Table 4 Cox proportional hazards analysis for cardiac events during follow-up

\begin{tabular}{|c|c|c|}
\hline & \multicolumn{2}{|l|}{ Univariate } \\
\hline & HR $(95 \% \mathrm{Cl})$ & $P$ value \\
\hline Age (per+1 year) & 1.05 (1.01 to 1.09$)$ & 0.04 \\
\hline BMI $\left(\right.$ per $\left.+1 \mathrm{~kg} / \mathrm{m}^{2}\right)$ & $1.47(0.79$ to 2.74$)$ & 0.25 \\
\hline Peak systolic BP $($ per $+5 \mathrm{~mm} \mathrm{Hg})$ & $0.49(0.25$ to 1.01$)$ & 0.05 \\
\hline NT-proBNP (per one log unit) & 1.51 (0.91 to 2.55) & 0.11 \\
\hline NYHA (per one class) & 1.45 (0.73 to 2.89) & 0.28 \\
\hline LV EF at rest (per+1\%) & 0.96 (0.91 to 1.02$)$ & 0.22 \\
\hline E/E' ratio at rest (per+1) & 1.15 (1.01 to 1.33$)$ & 0.04 \\
\hline LV EDV at rest $\left(p e r+1 \mathrm{~mL} / \mathrm{m}^{2}\right)$ & 1.01 (0.99 to 1.02$)$ & 0.12 \\
\hline LV ESV at rest $\left(\right.$ per $\left.+1 \mathrm{~mL} / \mathrm{m}^{2}\right)$ & 1.02 (0.99 to 1.03$)$ & 0.11 \\
\hline LV SV at rest $\left(p e r+1 \mathrm{~mL} / \mathrm{m}^{2}\right)$ & 1.00 (0.95 to 1.06$)$ & 0.82 \\
\hline$\Delta \mathrm{LV}$ SV (per+1 mL) & 0.92 (0.86 to 0.98$)$ & 0.01 \\
\hline$\Delta \mathrm{LV}$ ESV (per+1 mL) & $1.06(1.02$ to 1.10$)$ & 0.006 \\
\hline$\Delta$ LV EF (per+1\% absolute) & $0.83(0.75$ to 0.93$)$ & $<0.001$ \\
\hline Peak workload (per+1 Watt) & 0.98 (0.97 to 1.01) & 0.05 \\
\hline Peak VO ${ }_{2}($ per+1 $\mathrm{mL} / \mathrm{kg} / \mathrm{min})$ & $0.79(0.70$ to 0.91$)$ & $<0.001$ \\
\hline
\end{tabular}

$\Delta$ : difference between rest and after bodyweight squatting exercise.

$\mathrm{BMI}$, body mass index; $\mathrm{E} / \mathrm{E}^{\prime}$, ratio of pulsed-wave Doppler -derived early diastolic transmitral flow and tissue Dopplerderived early diastolic velocity obtained from the lateral mitral annulus; EDV, end-diastolic volume; EF, ejection fraction; ESV, end-systolic volume; LV, left ventricular; NT-proBNP, $\mathrm{N}$-terminal pro-B-type natriuretic peptide; NYHA, New York Heart Association; peak $\mathrm{VO}_{2}$, peak oxygen consumption; SV, stroke volume.

were not different as summarised in table 3. Peak $\mathrm{VO}_{2}$ correlated strongest with ESV and LV EF after squatting EX followed by $\mathrm{E} / \mathrm{E}$ ratio at rest $(\mathrm{r}=-0.61,0.56$ and -0.47 , $\mathrm{P}<0.001$ for all). Patients without an LV CR ( $\mathrm{n}=39,57 \%)$ were more likely to exhibit a reduced functional capacity $(\mathrm{n}=24,64 \%)$ than patients with an LV CR $(\mathrm{n}=5,7 \%)$ $(\mathrm{OR}=7.73, \mathrm{P}<0.001)$.

\section{Prognostic factors of clinical outcome}

Median follow-up time of patients was 725 days. Fourteen patients $(21 \%)$ developed adverse cardiac events as follows: five patients died of heart failure, one of sudden cardiac death and the remaining eight patients were hospitalised due to worsening heart failure (see figure 1). Unadjusted Kaplan-Meier curves depict eventfree survival in patients with DCM stratified by functional capacity and by LV CR after repetitive squatting EX in figure 2. The presence of LV CR had similar sensitivity and specificity ( 0.97 and 0.51 ) as a preserved functional capacity $(0.95$ and 0.68$)$ to detect a more favourable outcome in patients with DCM. Table 4 summarises the results of univariate Cox proportional hazard analysis of factors related to the development of cardiac events. Multivariate analysis showed that neither resting echocardiographic parameters (age, LV EF and E/E') nor 


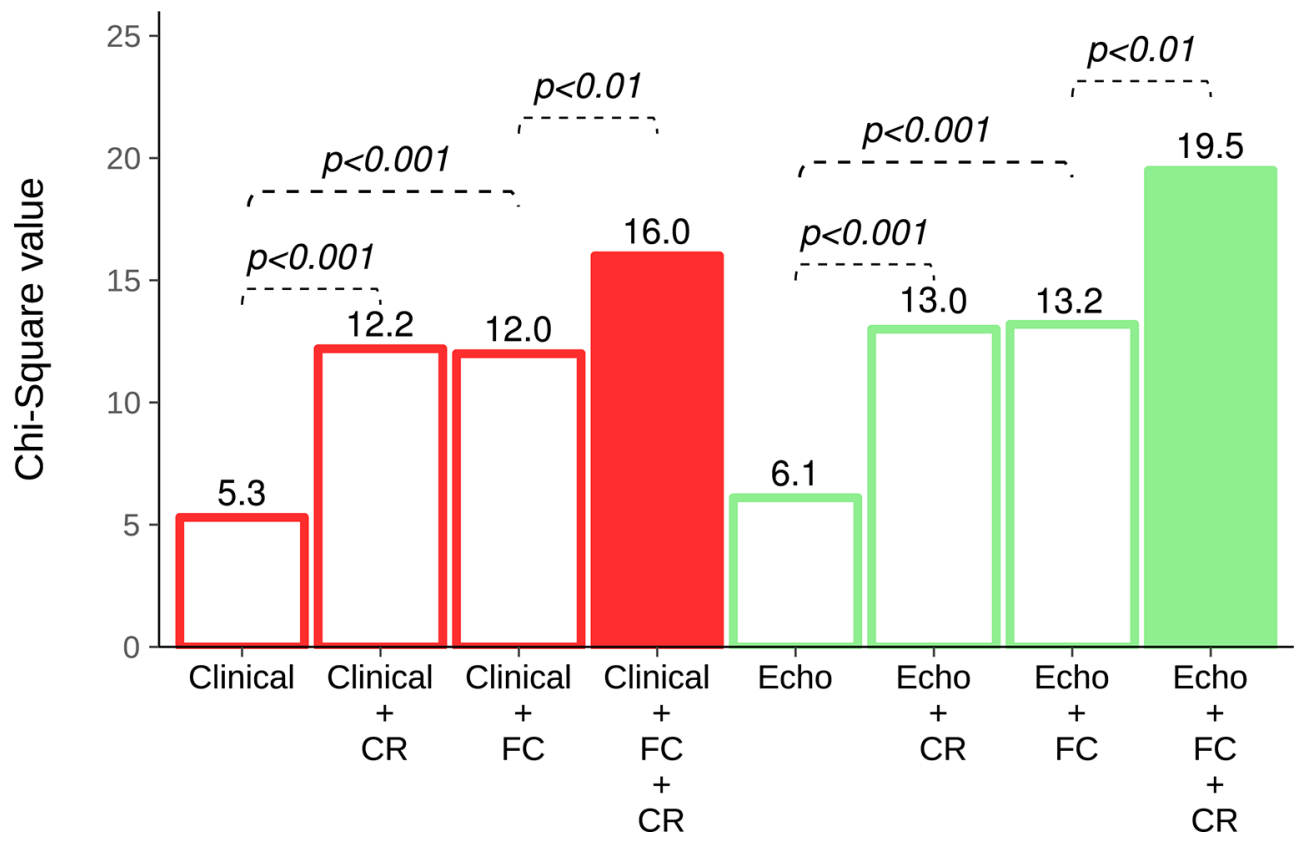

Figure 3 Incremental predictive value of CR after repetitive squatting exercise and FC from cardiopulmonary testing exercise results over clinical (red) and echocardiographic (green) information by likelihood ratio analysis expressed as $\chi^{2}$ values (y-axis) for prediction of adverse clinical events. Basal clinical model (first red column) consisted of age, NT-proBNP levels and NYHA functional class, while basal echocardiographic model (first green column) consisted of age, E/E' ratio and LV EF obtained at rest. $\mathrm{CR}$, contractile reserve; E/E', ratio of pulsed-wave Doppler-derived early diastolic transmitral flow and tissue Dopplerderived early diastolic velocity obtained from the lateral mitral annulus; EF, ejection fraction; FC, functional capacity; LV, left ventricular; NT-proBNP, N-terminal pro-B-type natriuretic peptide; NYHA, New York Heart Association.

clinical data (NT-pro BNP and NYHA) alone were independently associated with cardiovascular events $\left(\chi^{2}=5.35\right.$, $\mathrm{P}=0.14$ and $\left.\chi^{2}=6.07, \mathrm{P}=0.11\right)$. Adding information about $\mathrm{LV}$ CR to peakVO $\mathrm{V}_{2}$ and to main echocardiographic and clinical parameters yielded an incremental improvement in outcome prediction as depicted in figure 3. A summary of the multivariate analysis is found in online Table $\mathrm{S} 1$ of supplementary material. As expected, stratifying patients with DCM by the occurrence of cardiac events showed that these patients exhibited a deteriorating LV EF after repetitive squatting EX at baseline compared with those patients without cardiac events as depicted in figure 4 . Furthermore, baseline peak $\mathrm{VO}_{2}$ was significantly lower in patients with than without events during follow-up $(16 \pm 3$ vs $23 \pm 6 \mathrm{~mL} / \mathrm{Kg} / \mathrm{min}, \mathrm{P}<0.001)$.

\section{Intra-observer reliability}

Intraclass correlation coefficient (ICC) in the measurement of resting LV EDV and ESV was 0.98 and 0.97 , respectively, corresponding to LV EF of 0.97 ( $\mathrm{P}<0.001$ for all). Again after repetitive squatting EX, ICG values were as follows: $0.98,0.98$ and $0.97(\mathrm{P}<0.001$ for all $)$.

\section{DISCUSSION}

This study evaluated the prognostic role of LV CR after repetitive squatting EX by means of 3D echocardiography in patients with DCM. The major findings of this study are as follows: (1) repetitive squatting EX has the ability to disclose LV CR in patients with DCM and in healthy persons (figure 4); (2) absence of LV CR after repetitive squatting EX predicts future cardiac events (figure 2) similarly as a reduced functional capacity by CPET (figure 3); (3) it is superior than resting LV EF or clinical baseline characteristics and (4) LV CR assessed by repetitive squatting-standing EX is an additional diagnostic tool to CPET in order to discriminate patients at higher risk (figure 3 ).

Both, determination of functional capacity by CPET and assessment of LV CR by stress echocardiography are indispensable tools for differentiating adverse prognoses in patients with heart failure. ${ }^{3-4} 17-19$ CPET is regarded the clinical gold standard in the evaluation of patient's EX ability with peak $\mathrm{VO}_{9}$ as one of the main markers of functional performance. ${ }^{15}$ Traditionally stress echocardiography is performed with bicycle EX or with inotropic drugs in order to determine $\mathrm{LV} \mathrm{CR},{ }^{5}$ but it is labour intensive or not everywhere accessible in daily practice. Both peak $\mathrm{VO}_{2}$ and LV CR rely on increasing cardiac output due to an augmented $\mathrm{LV} \mathrm{SV}^{6}$ which is dependent on the decrease of LV ESV. ${ }^{20}$ Indeed, we could demonstrate that improving peak $\mathrm{VO}_{2}$ was strongly related to a concomitant decrease of LV ESV and increase of LV EF after squatting EX. Furthermore, LV CI adapted after EX with augmentation of LV SV and heart rate in order to fulfil blood/oxygen demand which is in line with the definition of CR. ${ }^{21}$ Patients with a decrease of LV $\mathrm{SV}$ and EF exhibited for instance a reduced functional capacity disclosing an insufficient rise of CR (figure 4). Repetitive squatting performed as a dynamic EX engages multiple muscle groups and provides positive inotropic 

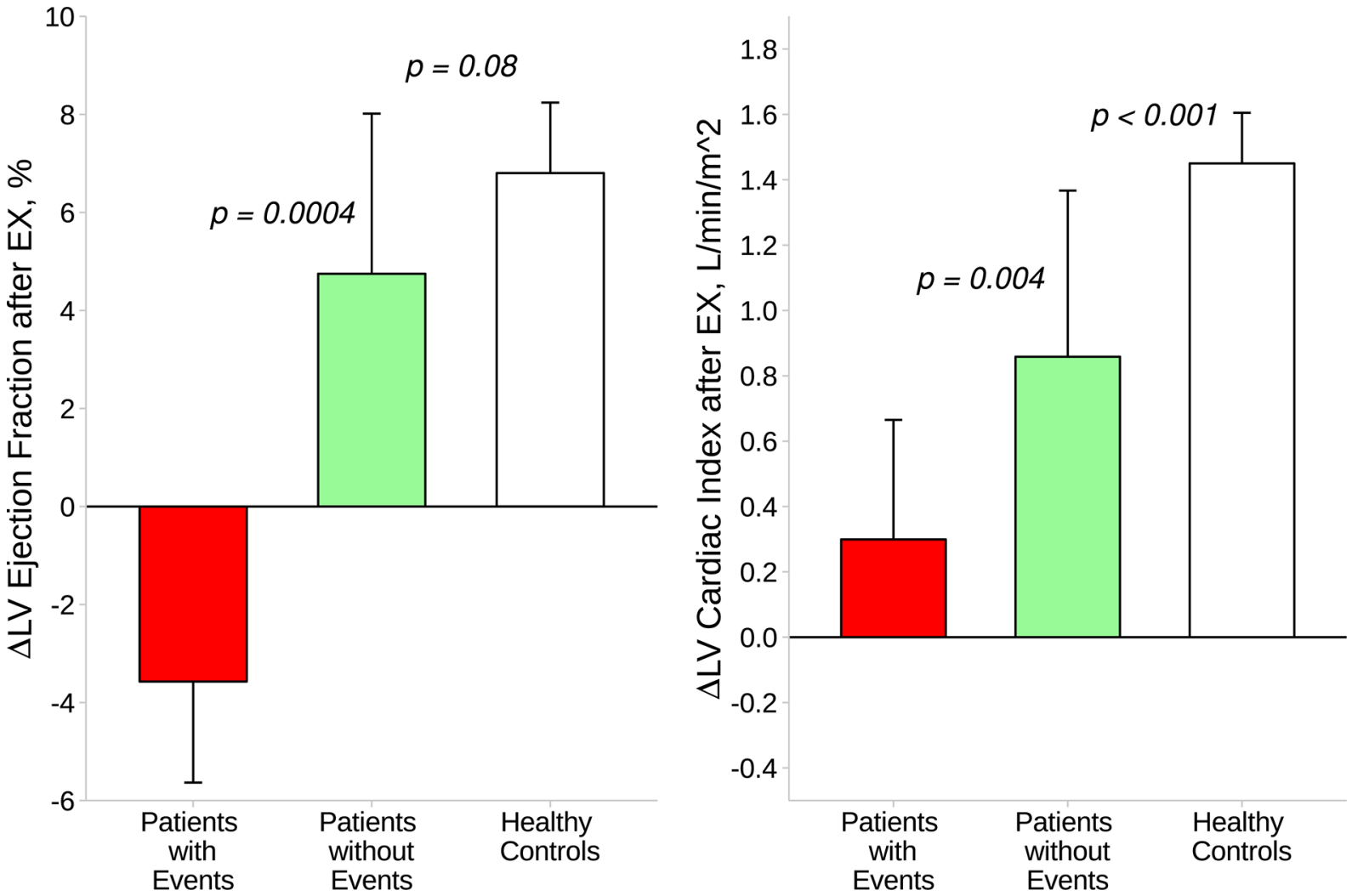

Figure 4 Changes $(\Delta)$ of left ventricular (LV) ejections fraction (left) and $\Delta$ cardiac index (right) after repetitive squatting exercise in patients with (red) and without (green) events compared with healthy controls (white). Data expressed as median with IQR. P values indicate significance levels for differences between groups assessed by appropriate test. EX, exercise.

and chronotropic effects on the healthy heart. ${ }^{1022}$ In this study, we clearly demonstrated that repetitive squatting EX has the ability to disclose an LV CR in patients with DCM and in healthy persons.

The determination of LV EF at rest remains routine practice to guide patient's therapy, although over the last decade the scientific community has given up on the idea that it could be the exclusive marker of functional capacity $^{1423}$ or of clinical prognosis. ${ }^{2425}$ Therefore, it should be important to evaluate LV functional response under dynamic conditions by use of EX or pharmacological stress that otherwise would be missed at rest. An all-accessible method of stress echocardiography in order to determine LV CR could enrich the daily armamentarium of cardiologists identifying patients with heart failure at higher risk for cardiac events. Indeed, as to our knowledge for the first time, our study demonstrates that the presence of LV CR after simple squatting EX is similarly predictive of a favourable clinical outcome in patients with DCM as the presence of a preserved functional capacity determined by CPET (figure 2). Both patients with and without a LV CR showed similar clinical and echocardiographic baseline characteristics at rest (tables 1 and 2). However, patients without LV CR developed a concomitant dilation of $\mathrm{LV}$ EDV and impairment of peak $\mathrm{VO}_{2}$ after $\mathrm{EX}$ which is indicative of a more progressive disease stage in these subjects. ${ }^{223}{ }^{26}$ Increase of preload after EX would induce myocardial prestretch with augmentation of myocardial contraction, for instance improving LV emptying in healthy hearts. However, in the context of per se reduced cardiac contractility ${ }^{27}$ with loss of contractile units augmented preload may induce elevated systolic wall stress and decrease lusitropy which in turn causes a drop in cardiac output due to a afterload/preload mismatch via a dysfunctional Frank-Starling mechanism in systolic heart failure. ${ }^{28}$ Indeed, our results underpin that LV CR after squatting EX is a superior predictor of outcome than resting echocardiographic parameters or other clinical characteristics (figure 3). These findings are in agreement with previous stress echocardiography studies $^{12} 1920 \square$ in which the absence of CR was a strong determinant of adverse outcome in patients with systolic heart failure. Our study clearly shows that a repetitive squatting-standing performed as a stress EX is an additional diagnostic tool to CPET in order to discriminate patients at higher risk.

\section{LIMITATIONS}

There are certain limitations to this prospective observational pilot study. First, this single-centre study was performed within a mid-term follow-up time and the diagnosis of heart failure was made $<6$ months. Second, the number of events in our study group was limited. Our patients with DCM exhibited concomitant elevated LV filling pressures at rest (ie, E/E' and NT-proBNP), 
but are considered as compensated and of lower risk for cardiac events. ${ }^{29}{ }^{30}$ This circumstance did not allow us to adjust the variables in a full multivariable model. Further studies with a broader collective of patients with systolic heart failure are needed to validate the present findings. Third, 3D echocardiography is limited by its relative low temporal resolution. However, we demonstrated good reproducibility of $\mathrm{LV}$ dimensions and $\mathrm{EF}$ at rest as well as after repetitive squatting EX. Furthermore, we are confident that the ongoing development in echocardiographic imaging will augment the usability of this method in order to facilitate further studies.

\section{CONCLUSION}

The results of this study demonstrate that a fast stress test by repetitive squatting EX reveals the crucial difference between heart failure patients with apparently similar stable clinical conditions at high risk and those at low midterm risk. LV CR assessed with 3D echocardiography and with repetitive squatting predicts cardiac events about as functional capacity assessed by CPET enriching the daily armamentarium of cardiologists.

Contributors All authors have contributed to this investigation as follows: study concept and proposal (KMS and SU: both authors contributed equally), data acquisition (KMS, SU, MR, CP, SQ, SK, KI), data analysis (KMS, SU, MR), drafting of manuscript (KMS, SU,RHS, SJ) and critical revisions of the manuscript (all authors).

Funding This research received no grant from any funding agency in the public or commercial sectors.

Competing interests None declared.

Ethics approval Institutional Review Board of Techinical University Dresden, Medical Faculty.

Provenance and peer review Not commissioned; externally peer reviewed.

Data sharing statement № additional data are available.

Open Access This is an Open Access article distributed in accordance with the Creative Commons Attribution Non Commercial (CC BY-NC 4.0) license, which permits others to distribute, remix, adapt, build upon this work non-commercially, and license their derivative works on different terms, provided the original work is properly cited and the use is non-commercial. See: http://creativecommons.org/ licenses/by-nc/4.0/

(c) Article author(s) (or their employer(s) unless otherwise stated in the text of the article) 2018. All rights reserved. No commercial use is permitted unless otherwise expressly granted.

\section{REFERENCES}

1. Matsumoto $\mathrm{K}$, Tanaka $\mathrm{H}$, Onishi $\mathrm{A}$, et al. Bi-ventricular contractile reserve offers an incremental prognostic value for patients with dilated cardiomyopathy. Eur Heart J Cardiovasc Imaging 2015;16:1213-23.

2. Naqvi TZ, Goel RK, Forrester JS, et al. Myocardial contractile reserve on dobutamine echocardiography predicts late spontaneous improvement in cardiac function in patients with recent onset idiopathic dilated cardiomyopathy. J Am Coll Cardiol 1999;34:1537-44.

3. Keteyian SJ, Patel M, Kraus WE, et al. Variables Measured During Cardiopulmonary Exercise Testing as Predictors of Mortality in Chronic Systolic Heart Failure. J Am Coll Cardiol 2016;67:780-9.

4. Francis DP, Shamim W, Davies LC, et al. Cardiopulmonary exercise testing for prognosis in chronic heart failure: continuous and independent prognostic value from VE/VCO(2)slope and peak VO(2). Eur Heart J 2000;21:154-61.

5. Lancellotti P, Pellikka PA, Budts W, et al. The clinical use of stress echocardiography in non-ischaemic heart disease: recommendations from the European Association of Cardiovascular
Imaging and the American Society of Echocardiography. Eur Heart $J$ Cardiovasc Imaging 2016;17:1191-229.

6. Weber KT, Kinasewitz GT, Janicki JS, et al. Oxygen utilization and ventilation during exercise in patients with chronic cardiac failure. Circulation 1982;65:1213-23.

7. Nesser HJ, Mor-Avi V, Gorissen W, et al. Quantification of left ventricular volumes using three-dimensional echocardiographic speckle tracking: comparison with MRI. Eur Heart $J$ 2009;30:1565-73.

8. Kleijn SA, Aly MF, Terwee CB, et al. Reliability of left ventricular volumes and function measurements using three-dimensional speckle tracking echocardiography. Eur Heart J Cardiovasc Imaging 2012;13:159-68.

9. Pickett CA, Cheezum MK, Kassop D, et al. Accuracy of cardiac $\mathrm{CT}$, radionucleotide and invasive ventriculography, two- and three-dimensional echocardiography, and SPECT for left and right ventricular ejection fraction compared with cardiac MRI: a metaanalysis. Eur Heart J Cardiovasc Imaging 2015;16:848-52.

10. Foster C, Meyer K, Georgakopoulos N, et al. Left ventricular function during interval and steady state exercise. Med Sci Sports Exerc 1999;31:1157-62.

11. McMurray JJ, Adamopoulos S, Anker SD, et al. ESC guidelines for the diagnosis and treatment of acute and chronic heart failure 2012: The Task Force for the Diagnosis and Treatment of Acute and Chronic Heart Failure 2012 of the European Society of Cardiology. Developed in collaboration with the Heart Failure Association (HFA) of the ESC. Eur J Heart Fail 2012;14:803-69.

12. Thomas DE, Wheeler R, Yousef ZR, et al. The role of echocardiography in guiding management in dilated cardiomyopathy. Eur J Echocardiogr 2009;10:iii15-21.

13. Alaranta $\mathrm{H}$, Hurri $\mathrm{H}$, Heliövaara $\mathrm{M}$, et al. Non-dynamometric trunk performance tests: reliability and normative data. Scand $J$ Rehabil Med 1994;26:211-5.

14. Hasselberg NE, Haugaa $\mathrm{KH}$, Sarvari SI, et al. Left ventricular global longitudinal strain is associated with exercise capacity in failing hearts with preserved and reduced ejection fraction. Eur Heart $J$ Cardiovasc Imaging 2015;16:217-24.

15. Guazzi M, Adams V, Conraads V, et al. Clinical recommendations for cardiopulmonary exercise testing data assessment in specific patient populations. Eur Heart J 2012;33:2917-27.

16. Lee R, Haluska B, Leung DY, et al. Functional and prognostic implications of left ventricular contractile reserve in patients with asymptomatic severe mitral regurgitation. Heart 2005;91:1407-12.

17. Chase PJ, Kenjale A, Cahalin LP, et al. Effects of respiratory exchange ratio on the prognostic value of peak oxygen consumption and ventilatory efficiency in patients with systolic heart failure. JACC Heart Fail 2013;1:427-32.

18. Paraskevaidis IA, Ikonomidis I, Simitsis $P$, et al. Multidimensional contractile reserve predicts adverse outcome in patients with severe systolic heart failure: a 4-year follow-up study. Eur J Heart Fail 2017;19:846-61.

19. Wever-Pinzon O, Bangalore S, Romero J, et al. Inotropic contractile reserve can risk-stratify patients with HIV cardiomyopathy: a dobutamine stress echocardiography study. JACC CardiovasC Imaging 2011;4:1231-8.

20. Scrutinio D, Napoli V, Passantino A, et al. Low-dose dobutamine responsiveness in idiopathic dilated cardiomyopathy: relation to exercise capacity and clinical outcome. Eur Heart J 2000;21:927-34.

21. Otasevic P, Popovic ZB, Vasiljevic JD, et al. Head-to-head comparison of indices of left ventricular contractile reserve assessed by high-dose dobutamine stress echocardiography in idiopathic dilated cardiomyopathy: five-year follow up. Heart 2006;92:1253-8.

22. Franklin BA. Aerobic exercise training programs for the upper body. Med Sci Sports Exerc 1989;21:S149-57.

23. Sveric KM, Ulbrich S, Rady M, et al. Three-Dimensional Left Ventricular Torsion in Patients With Dilated Cardiomyopathy- A Marker of Disease Severity. Circ J 2017;81:529-36.

24. Sengeløv M, Jørgensen PG, Jensen JS, et al. Global Longitudinal Strain Is a Superior Predictor of All-Cause Mortality in Heart Failure With Reduced Ejection Fraction. JACC Cardiovasc Imaging 2015;8:1351-9.

25. Stanton T, Leano R, Marwick TH. Prediction of all-cause mortality from global longitudinal speckle strain: comparison with ejection fraction and wall motion scoring. Circ Cardiovasc Imaging 2009;2:356-64.

26. Paraskevaidis IA, Adamopoulos S, Kremastinos DT. Dobutamine echocardiographic study in patients with nonischemic dilated cardiomyopathy and prognostically borderline values of peak exercise oxygen consumption: 18-month follow-up study. J Am Coll Cardiol 2001;37:1685-91. 
27. Kobayashi M, Izawa H, Cheng XW, et al. Dobutamine stress testing as a diagnostic tool for evaluation of myocardial contractile reserve in asymptomatic or mildly symptomatic patients with dilated cardiomyopathy. JACC Cardiovasc Imaging 2008;1:718-26.

28. Schwinger $\mathrm{RH}$, Böhm $M$, Koch $A$, et al. The failing human heart is unable to use the Frank-Starling mechanism. Circ Res 1994;74:959-69.
29. Mullens W, Borowski AG, Curtin RJ, et al. Tissue Doppler imaging in the estimation of intracardiac filling pressure in decompensated patients with advanced systolic heart failure. Circulation 2009;119:62-70.

30. Stienen S, Salah K, Eurlings LW, et al. Targeting N-Terminal Pro-Brain Natriuretic Peptide in Older Versus Younger Acute Decompensated Heart Failure Patients. JACC Heart Fail 2016;4:736-45. 